\title{
An Optimal Control Approach for Texture Metamorphosis
}

\author{
Ilknur Kabul $^{1}$, Stephen M. Pizer ${ }^{1}$, Julian Rosenman ${ }^{1,2}$, and Marc Niethammer ${ }^{1}$ \\ ${ }^{1}$ Department of Computer Science, UNC Chapel Hill, NC, USA \\ \{ilknurk, pizer, mn\}@cs.unc.edu \\ ${ }^{2}$ Department of Radiation Oncology, UNC Chapel Hill, NC, USA \\ rosenmju@med.unc.edu
}

\begin{abstract}
In this paper, we introduce a new texture metamorphosis approach for interpolating texture samples from a source texture into a target texture. We use a new energy optimization scheme derived from optimal control principles which exploits the structure of the metamorphosis optimality conditions. Our approach considers the change in pixel position and pixel appearance in a single framework. In contrast to previous techniques that compute a global warping based on feature masks of textures, our approach allows to transform one texture into another by considering both intensity values and structural features of textures simultaneously. We demonstrate the usefulness of our approach for different textures, such as stochastic, semi-structural and regular textures, with different levels of complexities. Our method produces visually appealing transformation sequences with no user interaction.
\end{abstract}

Keywords: texture interpolation, metamorphosis, texture synthesis, image morphing, registration, optimal control

ACM CCS: I.3.7 [Computer Graphics]: Three-Dimensional Graphics and Realism—Colour, Shading, shadowing and texture; I.3.6 [Computer Graphics]: Methodology and Techniques

\section{Introduction}

Although image registration only deals with the alignment of a source to a target image, image metamorphosis also considers changes in image appearance allowing to compute transitions from a source to a target image. Consequentially, image metamorphosis has been of increasing importance in computer graphics as well as in medical imaging. It has been used to morph between faces, to mix multiple images and to accommodate for appearance changes in image registration in general.

To obtain an image metamorphosis requires the computation of point correspondences over time (a time-dependent warp field) as well as an optimal change of pixel intensity to allow for an exact match to the target image. This joint optimization problem has, for example, been studied in [TY05, Hol09] and is a natural extension of fluid flow registration methods which estimate time-dependent velocity fields to smoothly transform one image into another [BMTY05]. Figure 1 illustrates the difference between registration and metamorphosis. In contrast to key-framing, for cartoon-like images the image metamorphosis approach does not take into account kinematics, but computes the space deformation using image information (and possible feature channels) only.

Texture metamorphosis is a special case of image metamorphosis which is used to design new textures by interpolating between the input textures or to visualize the change of material on the objects by creating a smooth transition between two textures. Structure features and appearance of the interpolated textures should perceptually be between input texture pairs. Creating a meaningful and smooth transformation is a difficult problem. Transformation of the intensity values as well as the feature masks, such as edges and ridges, should have an effect in the computation of the warping to split or merge structural features. 

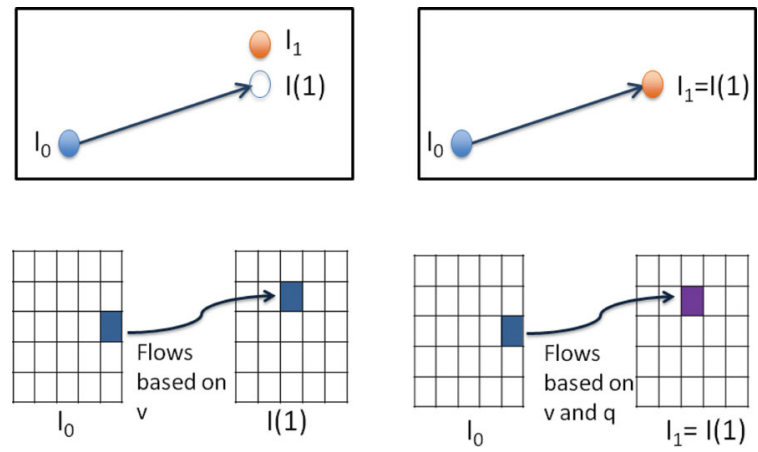

Figure 1: Comparison between registration and metamorphosism.

In this paper, we present a new texture metamorphosis approach considering structural and appearance transitions of textures in a single framework. Our method uses an optimal control framework (as proposed in [HZN09] for the image registration problem) and proposes a new numerical solution method which solves the metamorphosis problem as an initial value problem (and can therefore exploit a numerical solution strategy from a widely used fluid registration approach [BMTY05]). It is based on the large-displacementdiffeomorphic metric mapping (LDDMM) formulation of fluid registration [BMTY05, HZN09].

Our approach results in an intuitive solution method for texture metamorphosis and has a number of advantages compared to other approaches for texture metamorphosis.

1. It is designed to interpolate textures while considering appearance and features in a single framework. This removes the need for an additional blending or synthesis scheme.

2. It can easily accommodate different image information, such as colour or structural feature masks.

3. It can easily accommodate different measures of image/feature similarity, such as simple sum of squared differences, correlations or mutual information.

4. It is designed to be symmetric with respect to the two textures.

5. It is robust and automatic. It neither requires user input for feature correspondences nor the selection of transition functions.

6. It interpolates structural textures as well as stochastic and natural textures.

The remainder of the paper is organized as follows: Section 2 discusses related work on texture metamorphosis. Section 3 presents the overview of our method. Section 4 illustrates our optimal control based texture metamorphosis approach. How to add colour information is explained in Section 5. Section 6 details our texture metamor- phosis approach for structural textures. Section 7 presents results.

\section{Background}

Several approaches have been proposed to find the transformation from one image to another. Bar et al. [BJEYLW01] proposed a semi-morphing approach in which 50/50 blends of textures are created by performing texture analysis using wavelets. The first approach for morphing structural textures was presented in [LLSY02] using a pattern-based approach in which the user specifies landmarks and their correspondences for two textures. The landmarks are used to create a warp field and cross-dissolving accommodates texture appearance changes. This approach assumes that texture images are composed of similar texture patterns. In addition, it requires a large amount of user interaction. Later, in [WLi03], an algorithm for synthesizing texture mixtures from different input textures is presented. In this method, weight images are used to control how each source texture affects the result texture.

Zhang et al. [ZZV*03] synthesized progressively variant textures by using a texton mask as the feature image for input textures. In their method, first a mixed feature mask is generated by blending and binary-thresholding. Then this mixed feature mask is used to synthesize the mixed output texture. The main disadvantage of this approach is that it does not guarantee a smooth transformation from one image to another. In addition, an arbitrary number of images between two input images cannot be generated. Liu et al. [LLH04] propose a system to design novel textures by analysing and manipulating textures. Their method allows the user to manipulate texture features which can be used for alignment in morphing. User-defined feature maps are also used in [MZD05] to produce warps between textures. They also propose a histogram matching technique to avoid blurring of features. Their approach is only applicable to textures that are similar to each other. A level set advection method for morphing between texture features was proposed in [RLW*09]. This method first finds the warped texture features between two texture feature images. Then, a constrained texture synthesis approach is applied to generate texture with a given feature mask. Recently, a new patch-based approach was proposed in [RSK10]. In this approach, initially individual neighbourhoods of the input textures are locally warped and blended and then they are used to create new textures by an optimization algorithm. The disadvantage of this approach is that it cannot handle materials with large differences in feature scales. In addition, since the warping is done using only feature information, it cannot handle textures that do not have clear, meaningful features.

The metamorphosis formulation that our approach builds on has been studied, for example, in [TY05, Hol09] and numerical solution methods have been proposed in [MY01] 
and [GY05]. This line of work has mostly been concerned with morphing grey-valued or colour images and has so far not dealt with the problem of texture morphing.

The use of optimal control formulations for image timeseries has been proposed in [BIK02, CL10, NHZ09]. In contrast to metamorphosis, these approaches address image deformations only, but not appearance changes. Furthermore, in contrast to our metamorphosis formulation and [NHZ09, HZN09], [BIK02, CL10] solve the associated transport equation for the image directly, which requires advanced numerical methods to avoid image blurring.

\section{Overview}

Given two texture images $I_{0}$ and $I_{1}$, we want to create interpolated images $I(t)$. Here, $t$ corresponds to time $t \in[0,1]$ under the constraint that $I(1)=I_{1}$ and $I(0)=I_{0}$, which enforces that the initial and the final texture images are matched exactly under metamorphosis.

Since there are infinitely many possible ways to morph image $I_{0}$ into $I_{1}$, the solution space needs to be constrained in a meaningful way. In metamorphosis, this is accomplished by formulating an optimization problem which seeks to determine a time-dependent velocity field $v$ and a time-dependent source term $q$ which are appropriately regularized.

In an optimal control setting, the metamorphosis optimization problem is equivalent to minimizing the following energy $E$ under a dynamic constraint for the image change.

$$
\begin{aligned}
E(v, q) & =\int_{0}^{1}\|v\|_{L}^{2}+\|q\|_{Q}^{2} \mathrm{~d} t \\
\text { subject to } \quad I_{t}+(D I) v & =q, I(0)=I_{0}, I(1)=I_{1} .
\end{aligned}
$$

Here, the control variables $v$ (the time and space-dependent velocity field) controls the image deformation (flow of image over time), $q$ controls the change in appearance over time and D denotes the Jacobian. $L$ and $Q$ denote norms to penalize $v$ and $q$. The deformation map can be computed from $v$.

The following constraints are imposed onto the optimization problem:

1. Image flow: This constraint is simply a transport equation with a source term $q$ and controls the flow of the image from $I_{0}$ to $I(t)$. Intuitively, it can be considered as a constraint which imposes that image intensity along a streamline of the velocity field $v$ changes over time only through $q$. For $q=0$, intensities will be constant along such a streamline (characteristic).

2. Initial and final constraints: To allow for texture morphing between two texture exemplars, the initial and the final condition for $I$ are fixed. This is the main difference to standard image registration where an inexact match of the warped source image to the target image is admis- sible. This requires determining the time and spatially dependent $q$ such that the exact match is achieved under the image flow constraint.

Solving the optimization problem in Equation (1) is challenging. We make use of an adjoint solution method as proposed in [HZN09] for image registration. Details of the solution method and its relation to the corresponding image-to-image registration approach are given in Section 4.

\section{Texture Metamorphosis}

Image registration involves finding a transformation which maps one image to another image as well as possible. In contrast to image metamorphosis, image registration is only concerned with determining this mapping and does not consider appearance changes. Therefore, perfect image matching is in most cases not achievable in image registration because of image noise, structural differences or appearance differences in the images.

In fluid flow registration, the deformation is achieved by flowing the image $I_{0}$ with respect to a time-dependent velocity field $v$. The fluid flow registration problem is highly complex, because the full spatio-temporal velocity field needs to be estimated. To make this problem computable, non-smoothness of the velocity field is penalized, to obtain spatially coherent registration solutions [BMTY05, HZN09]. In fact, by choosing an appropriate norm to penalize nonsmoothness diffeomorphic image transformations can be assured.

The energy to be minimized in LDDMM [BMTY05, HZN09] fluid flow registration is

$$
E(v)=\int\|v\|_{L}^{2} \mathrm{~d} t+\frac{1}{\sigma^{2}}\left\|I(1)-I_{1}\right\|^{2}
$$

subject to $I_{t}+(D I) v=0, I(0)=I_{0}$,

where $\|v\|_{L}^{2}=\langle L v, L v\rangle$ and $L$ is a differential operator to encourage smoothness of the velocity field, typically chosen of the form $L=-\alpha \nabla^{2}+\gamma$. Here, $\alpha$ encourages smoothness of the vector field. For instance, for large $\alpha$ strong deformations are discouraged and the interpolations looks like blending; $\gamma$ controls how much the overall travel distance of a particle is counted in the energy. Intuitively, a large $\gamma$ will favour small overall displacements over an accurate image match. $\sigma$ is a scalar which controls the trade-off between image match and transformation smoothness. Note that the only difference to the metamorphosis problem in Equation (1) is that the final constraint $I(1)=I_{1}$ has been replaced by an inexact matching term $\left\|I(1)-I_{1}\right\|^{2}$. See [HZN09] for details on how to solve such an optimization problem.

To allow for exact matching, the metamorphosis formulation (1) adds a control variable $q$ to the transport equation which controls the image intensity change and is penalized 
according to $\|q\|_{Q}^{2}$, where $\|q\|_{Q}^{2}=\langle Q q, Q q\rangle$ and $Q$ is a chosen differential operator. (Note that for an easy numerical solution the operator is chosen as $Q=i d$, the identity.) In Equation (1), the weighting parameters of the source term $q$ and the velocity term $v$ are set to the same value. Changing the weights of these terms would produce different results. For example, increasing the weight for the source term and decreasing the weight for the velocity term would shift the balance towards stronger deformation and less aggressive blending.

To solve this constrained optimization problem, we convert it to its unconstrained form by adding the dynamic constraint through a time and space-dependent Lagrangian multiplier $\lambda$. We add the final state condition through the Lagrangian multiplier $\tau$ and obtain the unconstrained energy

$$
\begin{aligned}
E(v, q, \lambda, I, \tau)= & \int_{0}^{1}\|v\|_{L}^{2}+\|q\|_{Q}^{2} \\
& +\left\langle\lambda, I_{t}+(D I) v-q\right\rangle \mathrm{d} t+\left\langle\tau, I(1)-I_{1}\right\rangle
\end{aligned}
$$

with respect to $v(x, t), q(x, t), I$ and the Lagrangian multipliers $\lambda(x, t), \tau(x)$.

For a candidate minimizer of the unconstrained energy, its variation with respect to $v, q, I, \lambda$ and $\tau$ need to vanish (Details of how the differential equations are solved is presented in the Appendix). Computing the first variation and rearranging terms yields

$$
\begin{aligned}
& \delta E(v, q, \lambda, I ; \mathrm{d} v, \mathrm{~d} q, \mathrm{~d} \lambda, \mathrm{d} I) \\
& =\int_{0}^{1}\left\langle 2 L^{\dagger} L v+\lambda(D I)^{T}, \mathrm{~d} v\right\rangle \\
& \quad+\left\langle 2 Q^{\dagger} Q q-\lambda, \mathrm{d} q\right\rangle+\left\langle I_{t}+(D I) v-q, \mathrm{~d} \lambda\right\rangle \\
& \quad+\left\langle-\lambda_{t}-\operatorname{div} v(\lambda v), \mathrm{d} I\right\rangle \mathrm{d} t+\left.\langle\lambda, \mathrm{d} I\rangle\right|_{0} ^{1}+\left\langle\mathrm{d} \tau, I(1)-I_{1}\right\rangle \\
& \quad+\langle\tau, \mathrm{d} I(1)\rangle .
\end{aligned}
$$

Since $\delta E$ needs to vanish for any $\mathrm{d} v, \mathrm{~d} q, \mathrm{~d} I, \mathrm{~d} \lambda$ and $\mathrm{d} \tau$, fulfilling the boundary conditions (i.e. zero boundary conditions for $\left.v, I(0)=I_{0}, I(1)=I_{1}\right)$ at optimality the following conditions need to hold:

1. Initial Condition: Sets the starting point for the images at time $t=0$.

$$
I(0)=I_{0}
$$

2. State Equation (Transport Equation): Flows the image forward to time $t=1$ using the velocity field $v$. The source term in the transport equation simply changes image appearance and thus makes the appearance and disappearance of structures in an image possible.

$$
I_{t}+(D I) v=q
$$

3. Final Conditions: Sets the final image and provides a final condition for the adjoint.

$$
\lambda(1)=2 q(1), \quad I(1)=I_{1} .
$$

4. Adjoint Equation (Scalar Conservation Law): Governs the dynamics of the adjoint (Lagrangian multiplier $\lambda$ ). Since the final condition $\lambda(1)$ is given by (3), this equation can be solved backward in time. Note that the adjoint corresponds to the concept of moments in physics [MTY06] and the scalar conservation law for the adjoint consequentially resembles the physical law of conservation of momentum.

$$
-\lambda_{t}-\operatorname{div}(\lambda v)=0
$$

5. Compatibility Condition:

$$
2 L^{\dagger} L v+\lambda(D I)^{T}=0, \quad 2 q-\lambda=0 .
$$

The first equation is used to compute the gradient of $E$ with respect to $v$ on the interval $t=[0$, 1]. From $\delta E(v, q, \lambda, I ; \mathrm{d} v, \mathrm{~d} q, \mathrm{~d} \lambda, \mathrm{d} I)=\int_{0}^{1}\left\langle 2 L^{\dagger} L v+\right.$ $\left.\lambda(D I)^{T}, \mathrm{~d} v\right\rangle \mathrm{d} t$, the gradient $\nabla_{v}^{L_{2}} E(v)=2 L^{\dagger} L v+$ $(D I)^{T} \lambda$ with respect to the $L_{2}$ norm or $\nabla_{v}^{V} E(v)=$ $2 v+\left(L^{\dagger} L\right)^{-1}\left((D I)^{T} \lambda\right)$ in the $V$-norm (induced by the operator $L)$ is obtained.

The second equation illustrates that $q$ is directly related to the adjoint $\lambda$ (subject to a potential smoothing operator $Q$ ). To simplify the problem, we assume that the appearance control is penalized by an $L_{2}$ norm which makes $Q=i d$ and the second compatibility condition becomes $2 q-\lambda=0$.

The optimality conditions reveal an interesting structure: the only difference to the image-to-image registration [HZN09] case are the source term $q$ in the transport equation (the forward model), the additional compatibility condition on $q$ as well as the final condition on $I$.

\subsection{Adjoint solution method}

An analytic solution can in general not be obtained from the optimality conditions for metamorphosis. Numerical solution methods have been proposed in [MY01] and [GY05]. In both cases, the metamorphosis problem is cast as a boundary value problem, subject to the fixed source and target images and the solution proceeds by alternating gradient solution schemes for the image values (implicitly accounting for the image source terms, $q$, in the transport equation) and for the velocity field.

Here, by exploiting the relation between $\lambda$ and $q$, we sidestep an iterative update of the image $I$ and only perform gradient descent with respect to the velocity field $v$. This approach results in an initial value problem which allows using a similar map-based solution as in [BMTY05], reveals the 
simple structure of the underlying optimality conditions and allows to easily integrate alternative image-matching terms (as they only affect the estimates for the velocity field through the final condition for the adjoint; see [HCF02] for computations of these final conditions). Although the use of maps is not strictly necessary for the solution of the optimization problem [HZN09], it is beneficial to avoid smoothing due to numerical dissipation effects.

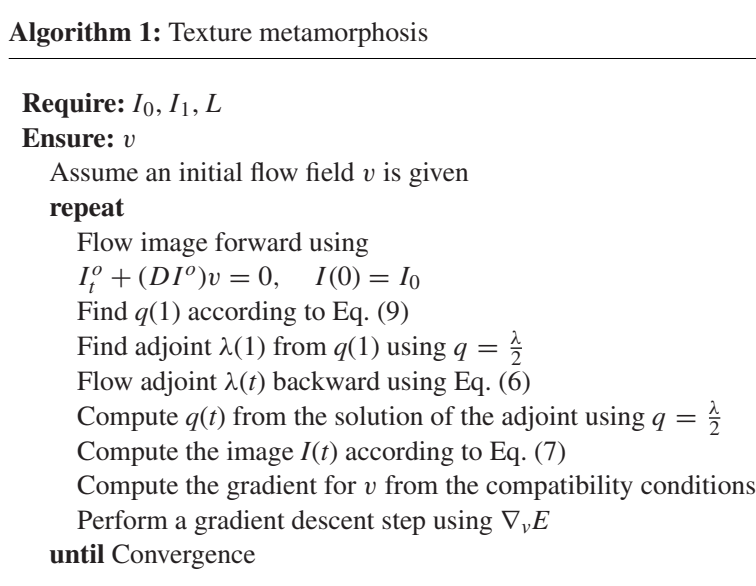

Specifically, given an estimate of the velocity field $v$, we compute the deformation maps $\Phi_{t}$ and $\Phi_{t}^{r}$ from $t=0$ to $t=$ 1 using

$$
\begin{gathered}
\Phi_{t}+(D \Phi) v=0, \quad \Phi(0)=i d, \\
-\Phi_{t}^{r}-\left(D \Phi^{r}\right) v=0, \quad \Phi^{r}(1)=i d
\end{gathered}
$$

where $i d$ denotes the identity map, $D$ denotes the Jacobian and $\Phi_{t}^{r}$ maps to $t=1$. This allows to replace the direct solution for $\lambda$ and $I$ by solutions of transport equations on the maps $\Phi$ and $\Phi^{r}$. The advantage of the map-based approach is that the maps are expected to be smooth (since the velocity fields are regularized according to $L$ ) and are therefore easier to propagate numerically.

To compute $\lambda$ at each time step according to the adjoint equation, $\lambda(1)$ is transported backwards in time using $\Phi^{r}(t)$. To conserve the values over time, the transformation is multiplied by $\left|D \Phi^{r}(t)\right|$, which corresponds to the amount of deformation the map has undergone.

$$
\lambda(t)=\left|D \Phi^{r}(t)\right| \lambda(1) \circ \Phi^{r}(t) .
$$

Using these maps to compute the solutions, $I(t)$ is slightly more complicated than in the standard image registration problem [BMTY05, HZN09] due to the source term $q$, which needs to be integrated along the characteristics (streamlines) of the velocity field to affect the overall intensity value. This can be expressed by adding the change of intensity to the

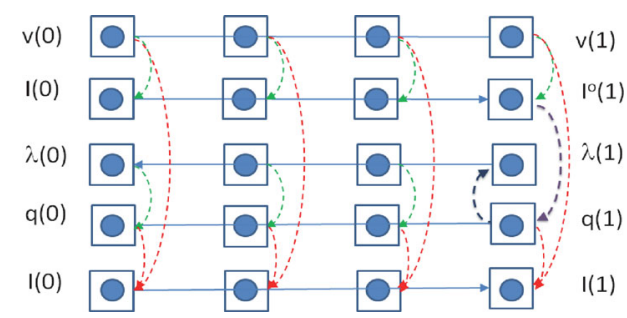

Figure 2: Optimal control solution framework for computing $I(t)$.

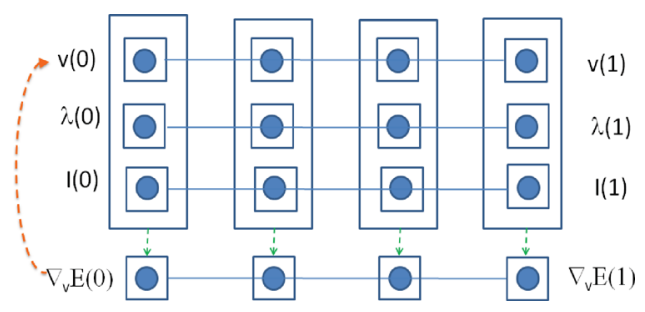

Figure 3: Optimal control solution framework for computing $\nabla_{v} E$.

transformed image $I_{0} \circ \Phi(t)$ as

$$
I(t)=I_{0} \circ \Phi(t)+\int_{0}^{t} q(\tau) \circ \Phi_{t, \tau} \mathrm{d} \tau,
$$

where $\Phi_{t, \tau}=\Phi(t) \circ \Phi^{-1}(\tau)$

which simply sums up all the $q$ contributions over time in the current reference coordinate system at time $t$.

Our proposed solution strategy builds on the observation that given a velocity field $v$ we can compute the final condition $\lambda(1)$ related to the source term $q$ which results in $I(1)=$ $I_{1}$ exactly. This is possible since we know from the optimality conditions that $q=\frac{\lambda}{2}$. Specifically, we observe that

$$
q(1)=\frac{I_{1}-I^{o}(1)}{\int_{0}^{1} \frac{1}{\left|D \Phi_{1, t}\right|} \mathrm{d} t}
$$

pointwise (The details of this equation are explained in the Appendix.). Given $q(1)$ we can compute $\lambda(1)$ and in turn $q(t)$. Here, $I^{o}(1)$ denotes the solution to the sourceless transport equation $I_{t}^{o}+\left(D I^{o}\right) v=0 ; I(0)=I_{0}$ using the given velocity field $v$.

The so-called adjoint solution method proceeds according to the Algorithm 1 which is illustrated in Figures 2 and 3. In effect, given an estimate of $v$ one (i) computes the solution to the sourceless transport equation, (ii) uses it to compute the final condition for $q$, (iii) which then allows the computation of $\lambda(t), q(t)$ and $I(t)$ and (iv) from which the gradient $\nabla_{v(t)} E$ can be computed. After taking a gradient descent step 


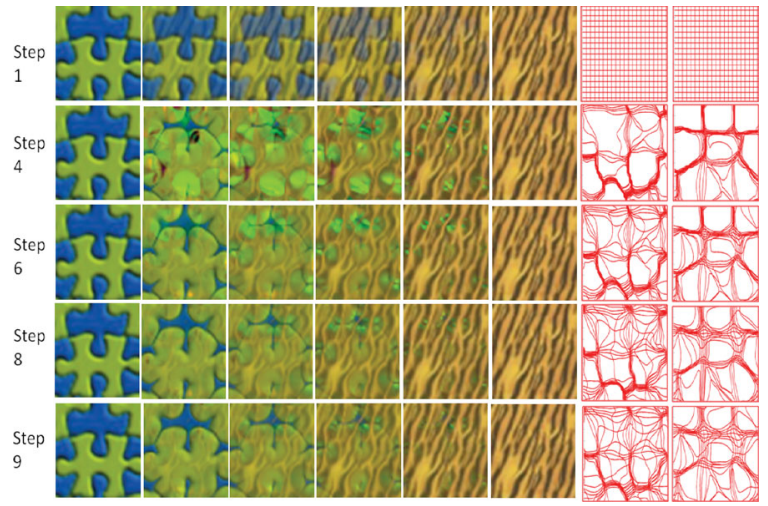

Figure 4: The texture sequences along with the deformation fields obtained in different steps of iteration.

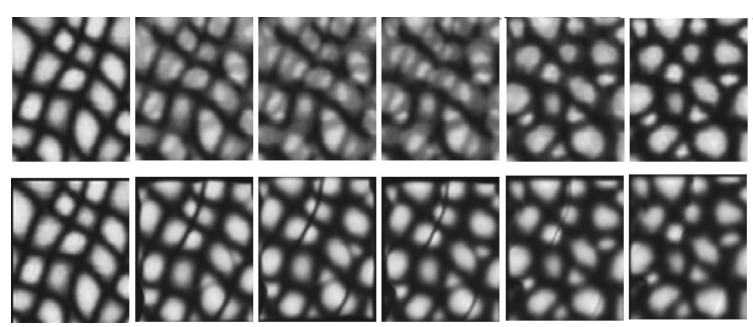

Figure 5: Metamorphosis from one texture to another using image intensities. Top row shows linear interpolation and bottom row shows our results.

with respect to the velocity field, these steps are repeated to convergence. Figure 4 shows the intermediate results along with the deformation fields in each step.

Figure 5 illustrates our metamorphosis results. Even though there is no control in the structural features of the textures, our approach can split and merge the patterns by only using the $q$ term.

\subsection{Combining forward and backward transition in metamorphosis}

According to the constraint $I(1)=I_{1}$, the morphed image $I$ (1) should always match the target image $I_{1}$. However, due to numerical inaccuracies, the constraint may not be satisfied exactly. To avoid this problem, we apply the solution method in the forward direction and the backward direction, which allows us to make the problem symmetric and to obtain exact matching by construction. Specifically, we use two appearance control terms $q_{F}$ and $q_{B}$ and weight them linearly. Here, $q_{F}$ controls the change in appearance from $I_{0}$ to $I_{1}$ and $q_{B}$ controls the change in appearance from $I_{1}$ to $I_{0}$. The modified energy function is

$$
\begin{aligned}
& E(v, q)=\int_{0}^{1}\|v\|_{V}^{2}+(1-t)\left\|q_{F}\right\|_{Q}^{2}+t\left\|q_{B}\right\|_{Q}^{2} \mathrm{~d} t \\
& \text { subject to } I_{F, t}+\left(D I_{F}\right) v=q_{F}, \\
& I_{B, t}+\left(D I_{B}\right) v=q_{B}, \quad I_{F}(0)=I_{B}(0)=I_{0}, \\
& I_{F}(1)=I_{B}(1)=I_{1},
\end{aligned}
$$

where $I_{F}(t)$ denotes the transformed image in the forward direction from $I_{0}$ to $I_{1}$ at time step $t$, and $I_{B}(t)$ refers to the transformed image in the backward direction from $I_{1}$ to $I_{0}$ at time step $t$.

The resulting optimality conditions are similar to the ones obtained previously (now for two sets of equations) except for the compatibility condition which becomes

$$
2 L^{\dagger} L v+(1-t) \lambda_{F}\left(D I_{F}\right)^{T}+t \lambda_{B}\left(D I_{B}\right)^{T}=0 .
$$

Note that this simply amounts to solving the problem twice (in opposite directions) while keeping only one velocity field that needs to be estimated. The gradient for the velocity field is then a linear combination of the gradients for the image solutions in the different directions and the image itself is recovered as

$$
I(t)=(1-t) I_{F}(t)+t I_{B}(t) .
$$

In what follows we assume that all equations are solved numerically in this way without explicitly writing down the resulting forward/backward equations.

\section{Metamorphosis for Colour Textures}

In texture metamorphosis, the traditional approach is to warp single intensity values which come either from the texture image or its feature mask. In our method, multiple channels can be easily integrated into the energy equation by adding $q$ terms for each channel. The energy equation becomes

$$
\begin{gathered}
E(v, q)=\int_{0}^{1}\|v\|_{L}^{2}+\sum_{i=1}^{n}\left\|q^{i}\right\|_{Q}^{2} \mathrm{~d} t \\
\text { s.t. } \quad I_{t}^{i}+\left(D I^{i}\right) v=q^{i}, I^{i}(0)=I_{0}^{i}, I^{i}(1)=I_{1}^{i},
\end{gathered}
$$

where $n$ is the number of channels that should be considered in the optimization framework. In our examples, we used the red, green and blue colour channels. For each channel independently, the same optimality conditions for metamorphosis are obtained (i.e. initial and final condition, transport equation and conservation law). The compatibility condition for $v$ that is used to compute the gradient changes becomes

$$
2 L^{\dagger} L v+\sum_{i=1}^{n} \lambda^{i}\left(D I^{i}\right)^{T}=0 .
$$




\section{Metamorphosis for Structured Textures}

Some of the textures have strong structural components, such as edges, ridges, etc. These features should be considered in metamorphosis to obtain visually pleasing results since the structural components may undergo topological changes such as splitting and merging. In this optimization framework, our aim is to simultaneously morph one image into another with respect to appearance (intensities) while aligning their feature masks (without appearance change). This can be accomplished by adding the feature mask as an inexact matching term (as in image registration) subject to a transport equation without source term. The energy equation to be minimized becomes

$$
\begin{aligned}
& E(v)=\int_{0}^{1}\|v\|_{L}^{2}+\|q\|_{Q}^{2} \mathrm{~d} t+\frac{1}{\sigma^{2}}\left\|F(1)-F_{1}\right\|^{2} \\
& \text { s.t. } \quad I_{t}+(D I) v=q, I(0)=I_{0}, I(1)=I_{1}, \\
& F_{t}+(F I) v=0, F(0)=F_{0} .
\end{aligned}
$$

This can be combined with multiple colour channels for metamorphosis as desired. Note that the feature image does not have a final state constraint.

Adding the inexact matching term results in optimality conditions for $F$ and its adjoint $\omega$ which are the same as for image registration [HZN09]. The compatibility condition for $v$ changes, respectively, to include the influence of the feature image (i.e. the feature image influence is added). All other optimality conditions remain the same as in Section 4.

$$
\begin{gathered}
F_{t}+(D F) v=0, \quad F(0)=F_{0}, \quad-\omega_{t}-\operatorname{div}(\omega v)=0, \\
2 L^{\dagger} L v+\lambda(D I)^{T}+\omega(D F)^{T}=0, \\
\omega(1)=\frac{2}{\sigma^{2}}\left(F(1)-F_{1}\right) .
\end{gathered}
$$

See Algorithm 2 for a description of the solution steps.

In Figure 6, the comparison of our approach with a texture synthesis based interpolation approach is shown. As can be seen in this figure, the intensity changes in the interpolated images are not smooth in the results obtained using the approach of $\left[\mathrm{RLW}^{*} 09\right]$. The bright regions inside the pattern are getting bigger first and then they are getting smaller to match with the target image. On the other hand, in our results, the bright regions move inside the patterns to generate the regions in the target image.

\section{Results}

Our technique is suited for regular, semi-regular and stochastic textures, since we perform the deformation and appearance change in a single framework. In Figure 7, we illustrate the results our approach generated using only image intensity information. As can be seen, our approach created textures which are sharp and detailed, even when it is hard
Algorithm 2: Feature-based texture metamorphosis
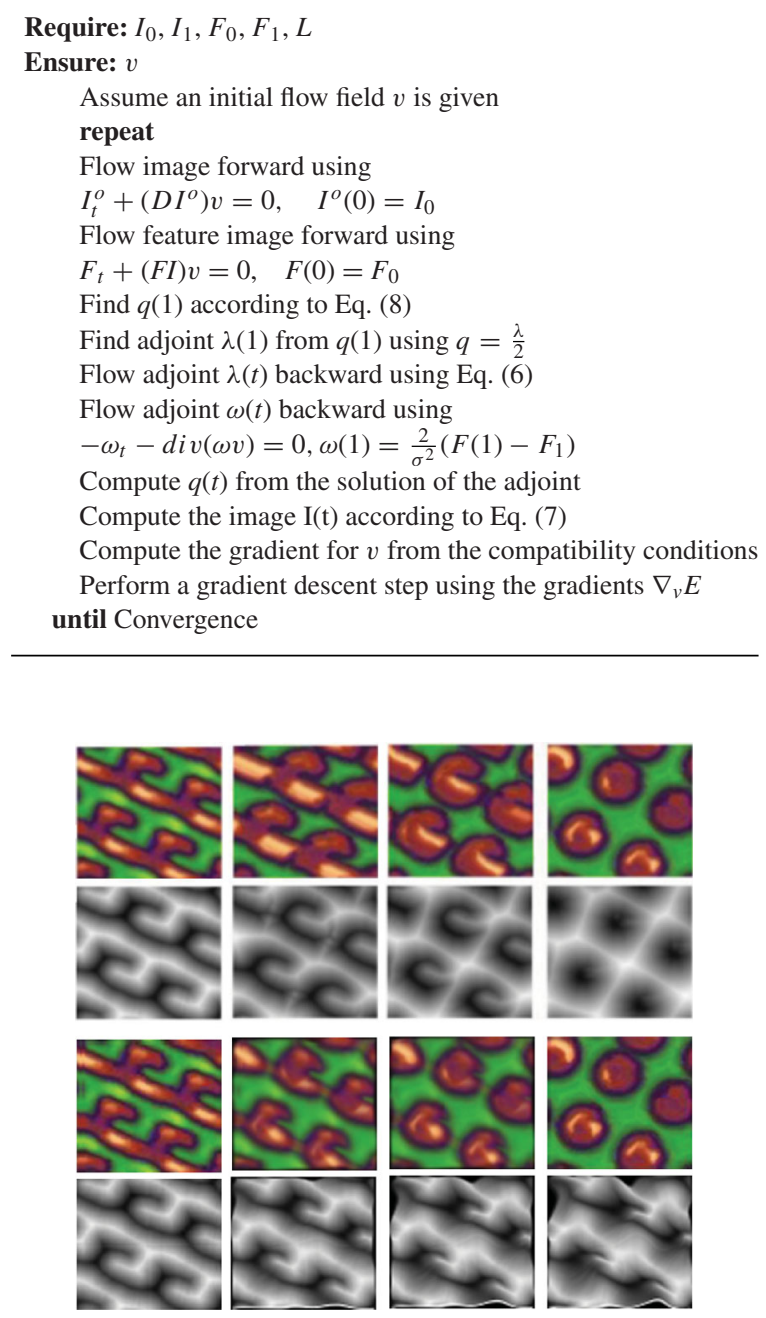

Figure 6: First and second rows show the results obtained using the approach in [RLW*09]. Third and fourth rows illustrate our results.

to define structures and the correspondences between them. Especially, the interpolation between pink flowers to white flowers created new flowers that have features present in both input images. The interpolated textures in this figure can be easily used in texture synthesis to generate textures on the surface of 3D models to visualize the change of materials over regions.

In Figure 8, we show the effect of using feature channels in metamorphosis. We obtained smooth and seamless transitions by propagating structural features and appearance by constraining the change in the feature along with intensity. Our results show that the addition of feature channels in metamorphosis provides more control over deformation.

(c) 2011 UNC Chapel Hill Computer Graphics Forum (c) 2011 The Eurographics Association and Blackwell Publishing Ltd. 

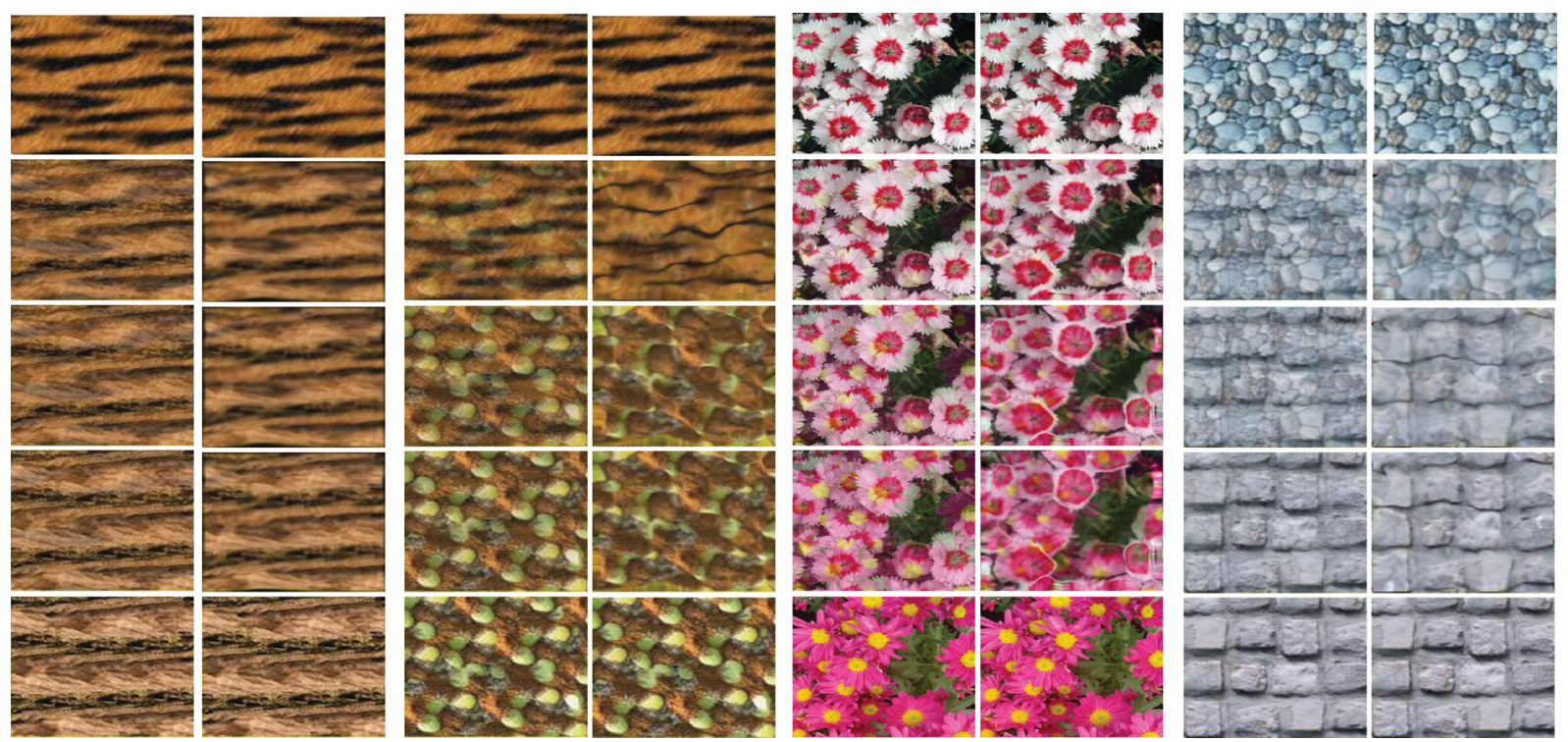

Figure 7: When meaningful features cannot be defined in the textures, such as natural textures, our metamorphosis can morph between textures by only considering appearance information. For each texture pair, first column shows the results with linear interpolation and second column illustrates the results with our approach.
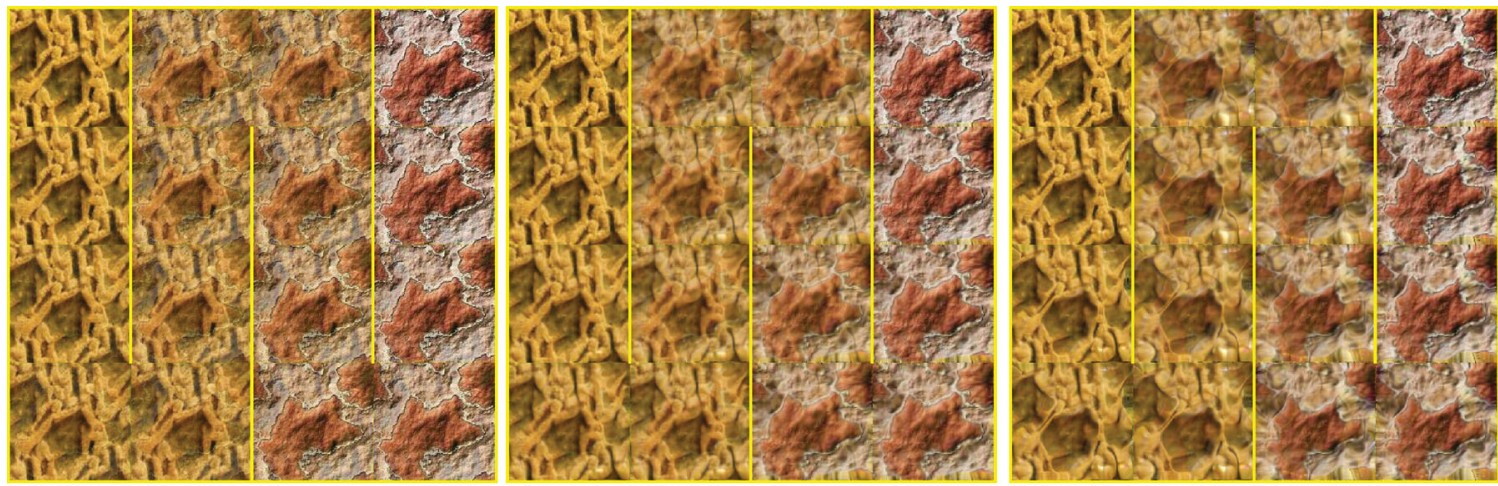

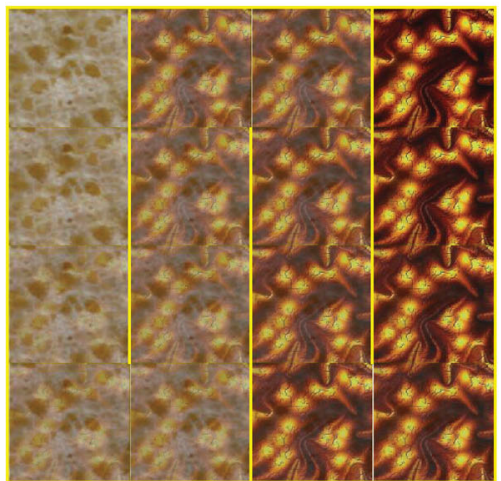

(a)

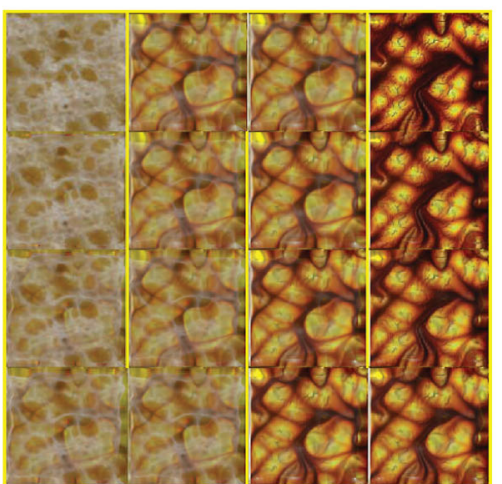

(b)

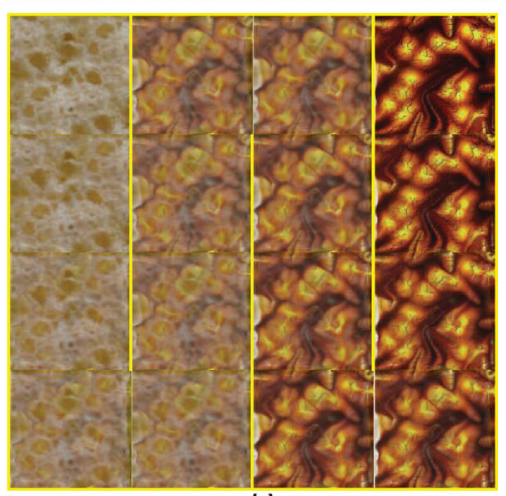

(c)

Figure 8: Including feature channel in metamorphosis changes the deformation. (a) Linear interpolation, (b) metamorphosis without using structural features and (c) metamorphosis using structural features. 

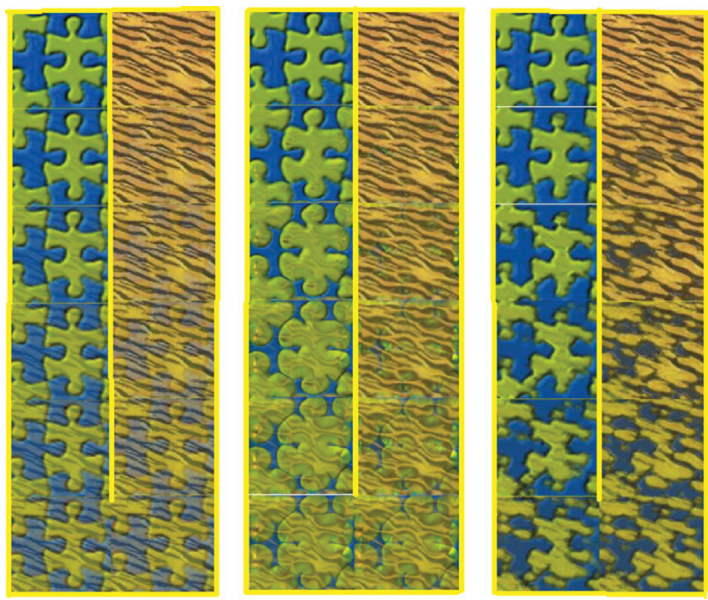

Figure 9: Comparison of interpolation results from [RLW*09] with our results. (Left-hand panel) Linear blending, (middle panel) our approach and (right-hand panel) results from [RLW*09].

In Figures 9 and 10, we show the comparison of our results with those presented [RLW*09]. In Figure 10, since there are no meaningful structural patterns in the input textures, we only used image information in the energy equation. Our approach created smooth transformations from the leopard texture to the dice texture by forming the dots on the dice from the spots on the leopard texture. In Figure 9, we used the structural feature information along with the image information to create interpolated textures. Since the method presented in [RLW*09] interpolates texton masks using an advection algorithm and then synthesizes interpolated textures constrained by these masks, the transition of the intensity values is not smooth and perceptually pleasing. In addition, the interpolated textures may contain new structures which are not available in either of the input textures. On the other hand, our approach deforms the structural features in the texture along with their intensity values. In Figure 11, we compared our results with those presented in [RLW*09] and [RSK10]. In this comparison, the textures in the middle of the sequence are different. Our approach seems to represent a nice compromise between blending of colour while at the same time achieving a good transition in terms of texture structure.

In Figure 12, we compare our results with the image registration method proposed in [HZN09]. We apply registration from $I_{0}$ to $I_{1}$ and $I_{1}$ to $I_{0}$, and combine the results obtained
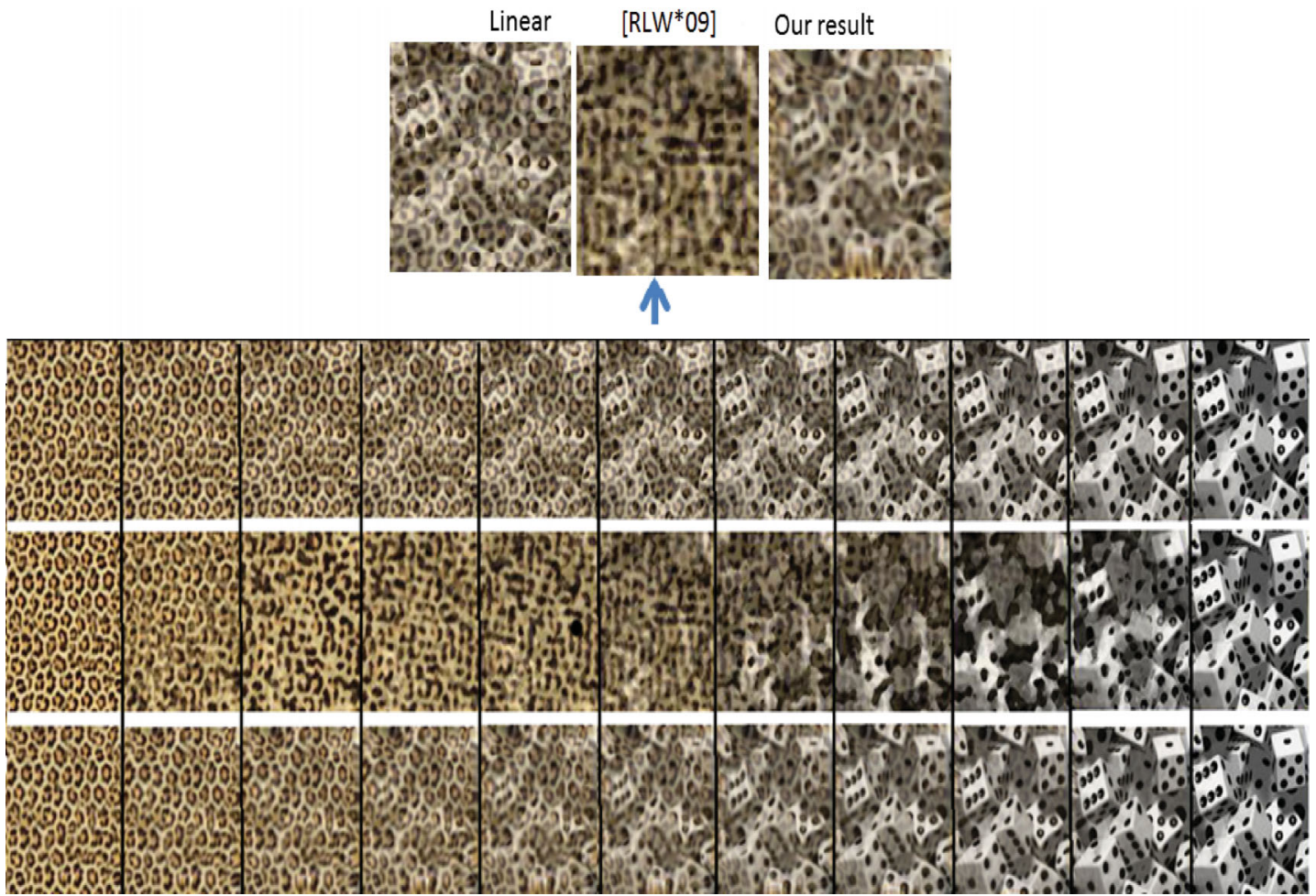

Figure 10: Comparison of interpolation results from [RLW*09] with our results. Top row shows the results obtained using linear interpolation, middle row illustrates the results from [RLW*09] and the bottom row shows the results obtained using our approach. 


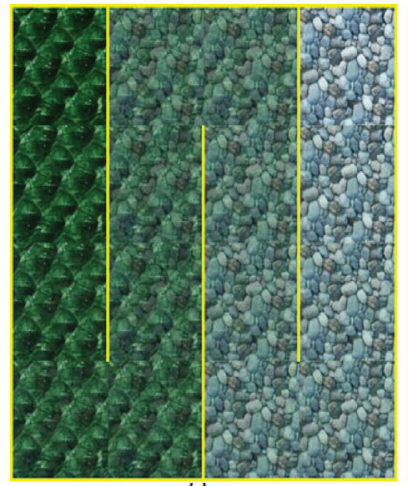

(a)

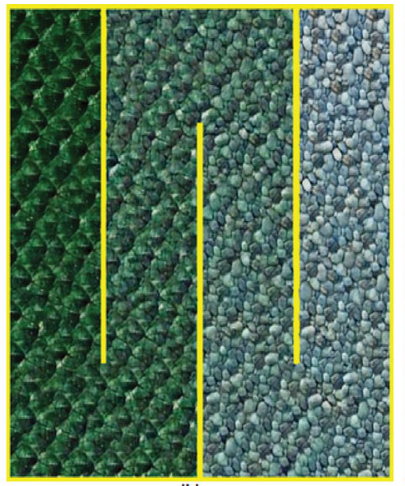

(b)

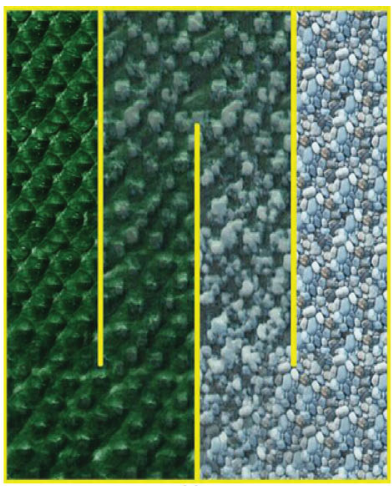

(c)

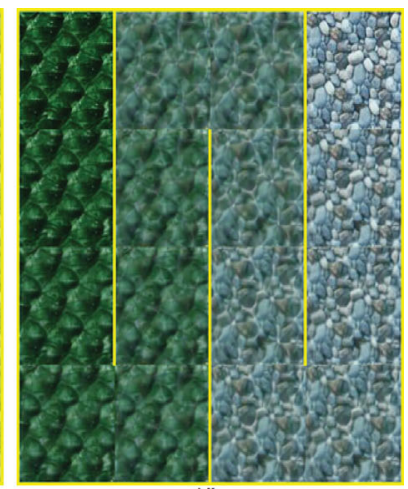

(d)

Figure 11: Comparison of results from with our results. (a) Linear blending, (b) results from [RSK10], (c) results from [RLW*09] and $(d)$ our approach.

from them using linear interpolation. As it is shown in the figures, the results obtained using registration and interpolation look more blurry. For the dots example in the first row, since the colour channels are treated independently in registration and there are no objects of corresponding colours in the source and target images, the algorithm shrinks the dots.

In Figure 13, we illustrate the effect of changing $\alpha$ in the differential operator $L$. Here, it can be seen that for large $\alpha$ strong deformations are discouraged, and the transformation becomes like blending. In Figure 14, some cases in which our approach fails are presented. For the textures that have many overlapping structural features, our approach sometimes fails to create valid intermediate textures.

The implementation of the proposed approach is done in Matlab and the source code is not optimized. The run time is usually in 5-10 minutes for $64 \times 64$ texture images. A GPU implementation of our approach would run within a few seconds.
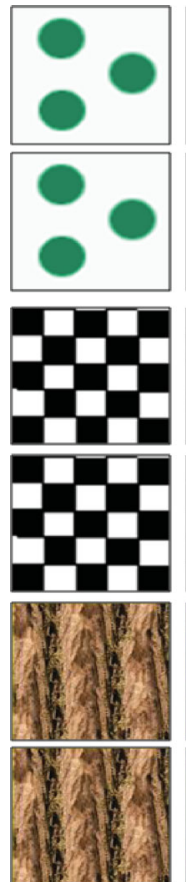
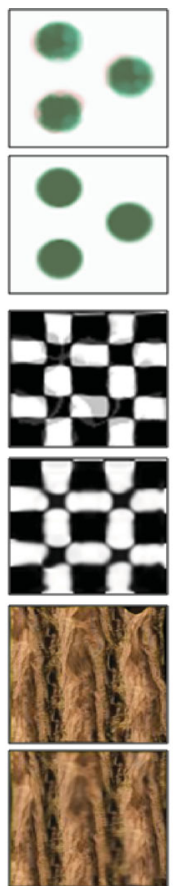
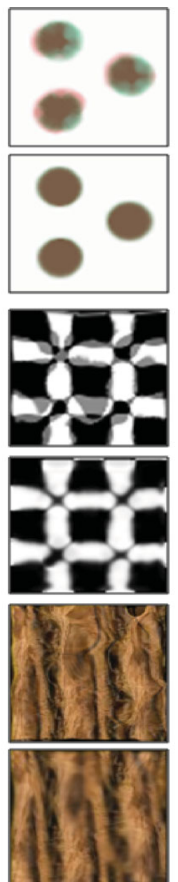
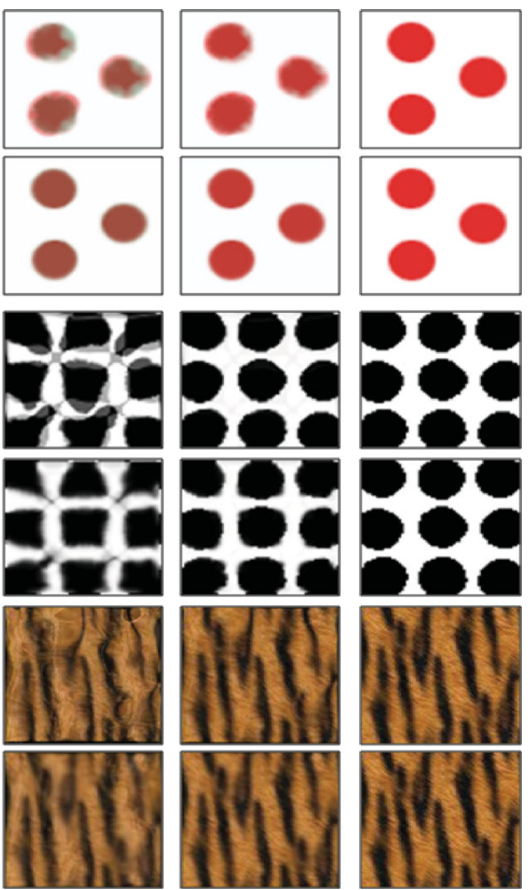

Figure 12: For each initial and target texture, top row shows the results obtained using registration and linear interpolation, and bottom rows show the results obtained using our metamorphosis approach. 


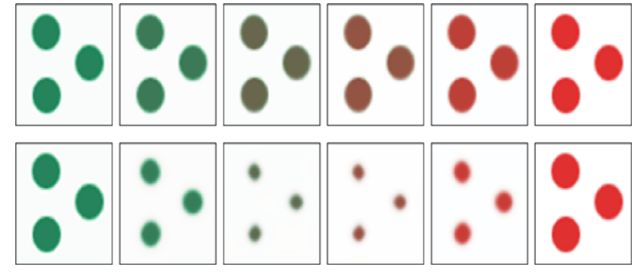

Figure 13: Top row illustrates results obtained by setting $\alpha$ is 0.01 , and bottom row illustrates results obtained by setting $\alpha$ is 0.001 .
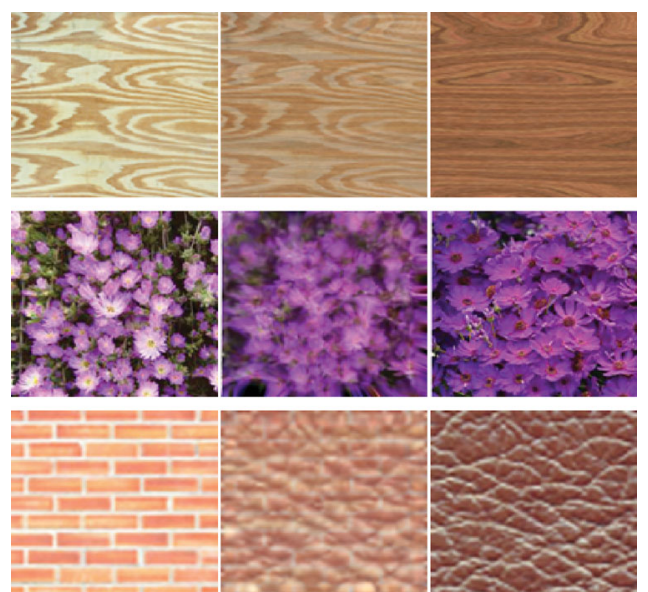

(a)

(b)

(c)

Figure 14: Failure cases. (a) Initial texture; (b) interpolated texture; (c) target texture.

\section{Conclusion and Future Work}

In this paper, we have presented a novel optimal control based texture metamorphosis approach, which warps one texture into another by considering the appearance and feature information in a single framework. In contrast to other techniques that are based on warping in texture features and texture synthesis (or blending) in creating interpolated textures, our approach can create consistent and visually pleasing interpolated images, even when it is not possible to define the features in the texture. In future work, we will integrate alternative features and landmarks into the energy equation to better control the transformation of the textures. Moreover, we only investigated the interpolation between two input textures. It would be interesting to design a new optimization framework with additional penalty terms and constraints to perform the interpolation between a number of input textures. In addition, metamorphosis between 3D solid textures would be interesting future work.

\section{Acknowledgements}

This material is based upon work supported by the National Science Foundation (NSF) under Grant No. EECS-0925875 and by the National Institutes of Health (NIH) under Grant Nos. (2 P41 EB002025-26A1, 1 R01 MH091645-01A1). Any opinions, findings, and conclusions or recommendations expressed in this material are those of the author(s) and do not necessarily reflect the views of NSF or NIH.

\section{Appendix}

\section{Optimality Conditions}

For a minimizer of energy, its variation with respect to $v, \lambda$, $I$ and $\gamma$ need to vanish. Computing

$$
\begin{aligned}
& \delta E(v, q, \lambda, I ; \mathrm{d} v, \mathrm{~d} q, \mathrm{~d} \lambda, \mathrm{d} I) \\
& =\left.\frac{\partial}{\partial \epsilon} E(v+\epsilon d v, I+\epsilon d I, \lambda+\epsilon d \lambda)\right|_{\epsilon=0}
\end{aligned}
$$

yields

$$
\begin{aligned}
\delta E(v, q, \lambda, I ; \mathrm{d} v, \mathrm{~d} q, \mathrm{~d} \lambda, \mathrm{d} I) \\
=\int_{0}^{1}\left\langle 2 L^{\dagger} L v, \mathrm{~d} v\right\rangle+\langle 2 q, \mathrm{~d} q\rangle \\
\quad+\left\langle\mathrm{d} \lambda, I_{t}+(D I) v-q\right\rangle \\
\quad+\left\langle\lambda,(\mathrm{d} I)_{t}+(D \mathrm{~d} I) v\right. \\
\quad+(D I) \mathrm{d} v-\mathrm{d} q\rangle \mathrm{d} t+\left\langle\mathrm{d} \tau, I(1)-I_{1}\right\rangle \\
\quad+\langle\tau, \mathrm{d} I(1)\rangle .
\end{aligned}
$$

Since

$$
\int_{0}^{1}\left\langle\lambda, \mathrm{d} I_{t}\right\rangle \mathrm{d} t=\int_{0}^{1}\left\langle-\lambda_{t}, \mathrm{~d} I\right\rangle \mathrm{d} t+\left.\langle\lambda, \mathrm{d} I\rangle\right|_{0} ^{1},
$$

and (by Green's theorem) $\langle\lambda,(D \mathrm{~d} I) v\rangle$ is equal to

$$
\langle-\operatorname{div}(\lambda v), \mathrm{d} I\rangle+\int_{\partial \Omega} \mathrm{d} I \lambda v \cdot \mathrm{d} S=\langle-\operatorname{div} v(\lambda v), \mathrm{d} I\rangle,
$$

we get

$$
\begin{aligned}
\delta E(v, q, \lambda, I ; \mathrm{d} v, \mathrm{~d} q, \mathrm{~d} \lambda, \mathrm{d} I) \\
=\int_{0}^{1}\left\langle 2 L^{\dagger} L v+\lambda(D I)^{T}, \mathrm{~d} v\right\rangle \\
\quad+\left\langle 2 Q^{\dagger} Q q-\lambda, \mathrm{d} q\right\rangle+\left\langle I_{t}+(D I) v-q, \mathrm{~d} \lambda\right\rangle \\
\quad+\left\langle-\lambda_{t}-\operatorname{div} v(\lambda v), \mathrm{d} I\right\rangle \mathrm{d} t+\left.\langle\lambda, \mathrm{d} I\rangle\right|_{0} ^{1} \\
\quad+\left\langle\mathrm{d} \tau, I(1)-I_{1}\right\rangle+\langle\tau, \mathrm{d} I(1)\rangle,
\end{aligned}
$$

assuming zero boundary conditions for $v$ and a known $I(0)=I_{0}$. Since $\delta E$ needs to vanish for any $\mathrm{d} v, \mathrm{~d} I, \mathrm{~d} \lambda$, fulfilling the boundary conditions of the problem, the optimality conditions are obtained. 


\section{Source Term}

To be able to compute the values for $q$ without implicitly obtaining them through a gradient descent on $I$, we can make use of the fact that for the $L_{2}$ norm penalizer, $q$ is directly related to the adjoint $\lambda$ and therefore also has to fulfil a scalar conservation law. Note that for a transport equation without a source term, values stay constant along the characteristics (streamlines) of the transport equation. With a source term the source values get integrated along the characteristic. Hence, along a characteristic

$$
I(t)=I(0)+\int_{0}^{t} q(t) \mathrm{d} t
$$

needs to hold. In particular, for our exact matching problem, we need to have

$$
I_{1}-I_{0}=\int_{0}^{1} q(t) \mathrm{d} t .
$$

However, representing everything in the coordinate frame of image $I_{1}$, we know that

$$
q(t)=\frac{1}{\left|D \Phi_{1, t}\right|} q(1),
$$

since it needs to fulfil a scalar conservation law. Assuming we discretize time into $n$ intervals spanning $[0,1]$ and assuming $q$ is piecewise constant over the time intervals

$$
\sum_{i=0}^{n-1} \frac{1}{\left|D \Phi_{1, t}\right|} q(1) \Delta t=I_{1}-I^{o}(1),
$$

which can be solved for

$$
q(1)=\frac{I_{1}-I^{o}(1)}{\sum_{i=0}^{n-1} \frac{1}{\left|D \Phi_{1, t}\right|} \Delta t} .
$$

In the limit as $\Delta t \rightarrow 0$ the sum becomes an integral and we get the desired expression (which holds pointwise)

$$
q(1)=\frac{I_{1}-I^{o}(1)}{\int_{0}^{1} \frac{1}{\left|D \Phi_{1, t}\right|} \mathrm{d} t} .
$$

expressed in the coordinate system of image $I_{1}$.

\section{References}

[BIK02] Borzì A., Ito K., Kunisch K.: Optimal control formulation for determining optical flow. SIAM Journal on Scientific Computing 24 (March 2002), 818-847.

[BJEYLW01] Bar-Joseph Z., El-Yaniv R., Lischinski D., Werman M.: Texture mixing and texture movie synthesis using statistical learning. IEEE Transactions on Visualization and Computer Graphics 7 (2001), 120-135.
[BMTY05] Beg M., Miller M., Trouvé A., Younes L.: Computing large deformation metric mappings via geodesic flows of diffeomorphisms. International Journal of Computer Vision 61, 2 (2005), 139-157.

[CL10] Chen K., Lorenz D. A.: Image sequence interpolation using optimal control. CoRR abs/1008.0548, (2010).

[GY05] Garcin L., Younes L.: Geodesic image matching: A wavelet based energy minimization scheme. In Lecture Notes in Computer Science (2005), vol. 3757, p. 349.

[HCF02] Hermosillo G., Chefd'hotel C., Faugeras O.: Variational methods for multimodal image matching. International Journal of Computer Vision 50, 3 (2002), 329-343.

[Hol09] Holm D.: Euler's fluid equations: Optimal control vs optimization. Physics Letters A 373, 47 (2009), 4354-4359.

[HZN09] Hart G. L., Zach C., Niethammer M.: An optimal control approach for deformable registration. In Proceedings of the Workshop on Mathematical Methods in Biomedical Image Analysis (MMBIA) (Miami, FL, USA, 2009).

[LLH04] LiU Y., Lin W.-C., HaYs J.: Near-regular texture analysis and manipulation. ACM Transactions on Graphics 23, 3 (2004), 368-376.

[LLSY02] Liu Z., Liu C., Shum H.-Y., Yu Y.: Pattern-based texture metamorphosis. In $P G$ '02: Proceedings of the 10th Pacific Conference on Computer Graphics and Applications (Washington, DC, USA, 2002), IEEE Computer Society, p. 184.

[MTY06] Miller M. I., Trouve A., Younes L.: Geodesic shooting for computational anatomy. Journal of Mathematical Imaging and Vision 24 (2006), 209228.

[MY01] Miller M. I., Younes L.: Group actions, homeomorphisms, and matching: A general framework. International Journal of Computer Vision 41, 1/2 (2001), 61-84.

[MZD05] Matusik W., Zwicker M., Durand F.: Texture design using a simplicial complex of morphable textures. ACM Transactions on Graphics 24, 3 (2005), 787-794.

[NHZ09] Niethammer M., Hart G. L., Zach C.: An optimal control approach for the registration of image time-series. In Proceedings of the Conference on Decision and Control (Shanghai, China, 2009), pp. 2427-2434.

[RLW*09] Ray N., LÉvy B., Wang H., Turk G., RunO Vallet B.: Material-space texturing. Computer Graphics Forum 28, 6 (2009), 1659-1669. 
[RSK10] Ruiters R., Schnabel R., Klein R.: Patch-based texture interpolation. Computer Graphics Forum 29, 4 (June 2010), 1421-1429.

[TY05] Trouve A., Younes L.: Metamorphoses through lie group action. Foundations of Computational Mathematics 5, 2 (2005), 173-198.
[WLi03] WeI Li: 2003: Texture synthesis from multiple sources. In Proceedings of the SIGGRAPH 2003 Sketches and Applications (San Diego, CA, USA, 2003).

[ZZV*03] Zhang J., Zhou K., Velho L., Guo B., Shum H.Y.: Synthesis of progressively-variant textures on arbitrary surfaces. ACM Transactions on Graphics 22, 3 (2003), 295-302. 